\title{
STUDY ON SUPER LONG DISTANCE SPACE TELEROBOTICS
}

\author{
Hiroaki Ujiie*, Fumio Yasumoto*, Kenji Ogimoto*, \\ Shigeoki Hirai**, Kazuo Machida**, Yujin Wakita**, \\ S.Peters***, and P.Backes*** \\ * Kawasaki Heavy Industries Ltd. 1 Kawasaki-cho Kakamigahara city, \\ ** Electrotechnical Laboratory AIST, MITI 1-1-4 Umezono Tsukuba city,
} Gifu Pref. 504 Japan Ibaraki Pref. 305 Japan

*** Jet Propulsion Laboratory, NASA, USA

\begin{abstract}
Telerobotics research for Super Long Distance has been underway between MITI of Japan and NASA JPL of United States. Foe this telerobotics, the limited amount of data transfer, communication delay and the uncertainty of robot environment will be the major issues. To overcome these problems, a telerobotic system, with a knowledge base for object handling, an environment model and a hybrid compliance/force control, has been constructed. With this system, a truss structure in Electrotechnical Lab. in Japan was successfully deployed from JPL in United States. The experimental set-up with built-in software and test results are described in this paper.
\end{abstract}

\section{INTRODUCTION}

In the field of construction, telerobotics have been studied to operate various rolling stocks and construction robots. These robots are intended to operate under the hazardous conditions. Kawasaki has been studying intelligent telerobotics under the supervision of Electrotechnical Laboratory(ETL), in which a robot on a super long distance is teleoperated.

Due to limited amount of data transfer and the uncertainty of the environment, a certain degree of robotic autonomy is required for the system. To realize the autonomy various types of knowledge bases for object handling, environmental models, intelligent monitoring scheme[4] have been proposed and furthermore a hybrid compliance/force control[6] has been implemented in the system to improve the performance. This system has been operated from JPL of NASA in USA to expand the deployable truss structure in ETL in Japan.

\section{TELEROBOTICS ELEMENTS}

Besides the limited amount of data transfer, the time delay and interrupt of communication, and the uncertainty of the environment, it is often the case to have primitive level of hardware such as controllers, sensors and monitoring devices, and software. Thus information for an ground operator will be insufficient. Even under these circumstances, a teleoperation system has to be effective and should be able to handle objects in definite manner. 


\subsection{DISTRIBUTED TELEROBOTICS [1]}

Dr.Machida et al proposed the distributed system, in which the robotic functions are distributed between robot site(Remote) and operator site(Local). The system described in this paper is developed closely following this distributed system. On Remote Site, the robot has a certain degree of autonomy and necessary skills, and the control system on Local Site has the intelligent processing function to support an operator under the limited amount of data transfer.

\subsection{KNOWLEDGE BASE FOR OBJECT HANDLING [4]}

To accommodate the autonomy in the remote system, the system has to have the knowledge base for object handling. To realize the above system, Dr. Hirai et al proposed the telerobotic system which includes a knowledge base for object handling and an environmental model. In the knowledge base, positions, conditions, shapes, and operation methods and procedures for objects are included, and states in a work area are described in the environmental model. The knowledge base manages and renews the environmental model according to the object handling. Furthermore, an operator can communicate with the knowledge base to modify the operation procedures.

By using the knowledge base, an operator on Local Site can command the robot by sending the task level commands instead of robot's actuator level commands,thus reduced the load on an operator and the amount of data transfer between Remote and Local Sites. The knowledge base is also used for the intelligent monitoring, the direct teaching, and the graphics simulator.

This knowledge base has been developed using EusLisp[11] which was developed to facilitate Object Oriented Language by ETL.

\subsection{INTELLIGENT MONITORING [4]}

Generally speaking, it is the best way to have camera views to confirm the operation on Remote Site.However, the field of view, focusing and zooming of a camera are usually controlled manually, and it is a time consuming operation to obtain a proper view of a work site for every task. To improve the situation described above, the following functions are incorporated in the system.

(1) Intelligent Monitoring

Finding the proper view of a monitoring camera for the intended tasks needs intelligent functions. Thus the function, to operate the camera autonomously according to the operation sequence using the knowledge base for object handling, is built in the system.

(2) Snapshot

Because of the limited amount of data transfer between the sites, it is practically impossible to send the camera view in real time. It was found that still views of critical scene would be enough to monitor work progress. Thus it was decided to send some snapshots for the end of each task to confirm the completion of the task.

(3) Sequencing of monitoring and operation

During the data transfer of images, the robot on Remote Site should not proceed to the next step. To guarantee the above operation, the sequencing of monitoring and operation was built in the system.

\subsection{GRAPHICS SIMULATION [5]}

Since the knowledge base manages the environmental model, it is possible to create graphics images using the updated environmental model. The graphics images thus created gives many 
useful information, and sometimes an operator can rely on the graphics images, especially when no other information is available for an operator. The following functions are built in this system, in relation with Graphics Simulator.

(1) Real-Time Graphics Simulation

Real view of Remote Site cannot be observed in real time on Local Site, because only the intermittent snapshots will be transferred to Local Site. However, supposing the start and end of a task and execution time are well defined and the task proceeds as planned, the graphics simulator can supply the real time images as the task is executed, namely a predictive display.

(2) Graphical Presentation of the Expected Task

It is useful to have the final image of an expected task, to prepare for the next step in advance. It was proposed to run the simulation on Remote Site according to the expected task command, and the result of the simulation was displayed graphically on Local Site.Thus an operator can see the results of the task command and feels confident with the sending the task command.

\subsection{DIRECT TEACHING MODE [5]}

In normal mode, the system is basically operated using task level commands. However, the system has to have the capability to cope with the unexpected trouble. For this purpose, it has the direct operation mode intervened by an operator, i.e. Direct Teaching Mode. Even in this mode, the knowledge base case be utilized to support an operator to handle the robot and the objects easily.

\subsection{HYBRID COMPLIANCE/FORCE CONTROL [6]}

It is often the case in which an environment and its model have the discrepancy, especially the positions and attitudes. It is difficult to match the environmental model exactly to the real world unless various types of sensors are available. In practice, a certain errors in positions and attitudes are acceptable and the special control scheme of a robot will be introduced.

Furthermore, there are some tasks in which soft contact with objects are required. To cope with these situations, an $6 \mathrm{DOF}$

force/torque sensor was attached to the wrist of the robot arm together with the hybrid compliance/force control program. Damping and rigidity were introduced through the compliance control to absorb the position and attitude errors, and pushing and pulling forces to objects were controlled by the force control."

\section{SYSTEM CONSTRUCTION [2][3][5]}

A system block diagram is shown in Fig.1, which is described in the chapter 2.

\subsection{LOCAL SITE SYSTEM}

There is a workstation with UNIX for the remote operation of the robot on Remote Site. The local site workstation is loaded knowledge base, and processes EusLisp, X-videos, and graphics simulator.

\subsection{COMMUNICATION LINE}

A communication line between local site in USA and remote site in JAPAN is connected by international ISDN line with TCP/IP protocol for transmittance and reception of commands and 
datum.

\subsection{REMOTE SITE SYSTEM}

Remote site system consists of a workstation, a VME controller, a robot system a camera system and a test bed.

Remote Site Workstation

There is a workstation with UNIX for the communication with the local site. And the workstation has a knowledge base such as a local site one.

VME controller

The VME controller manages remote site local area network between the workstation, the robot system and the camera system.

Robot System

The robot system consists of a remote robot and a robot controller. The remote robot is a KHI Js-10 anthropomorphic manipulator with $6 \mathrm{DOF}$. And there is a 6 axis force/torque sensor, a three finger gripper, a hand-eye camera, a laser pointer on the robot hand. The 6 axis force/torque sensor's signals are inputted to Hybrid Compliance/Force Control system. The three finger gripper grasps and releases an object. The hand-eye camera gets a image around the robot hand. The laser pointer which is set in the center of the three finger gripper projects a light point on a target surface.

Camera System

The camera system consists of a supervisor camera and a camera controller. The supervisor camera which is set on the camera table is controlled pan/tilt/zoom/focus by camera controller, and gets images of the remote site scene.

Test Bed

A test bed modeled space platform consists of a two-cell truss structure, Orbital Replaceable Unit(ORU), and ORU holder.

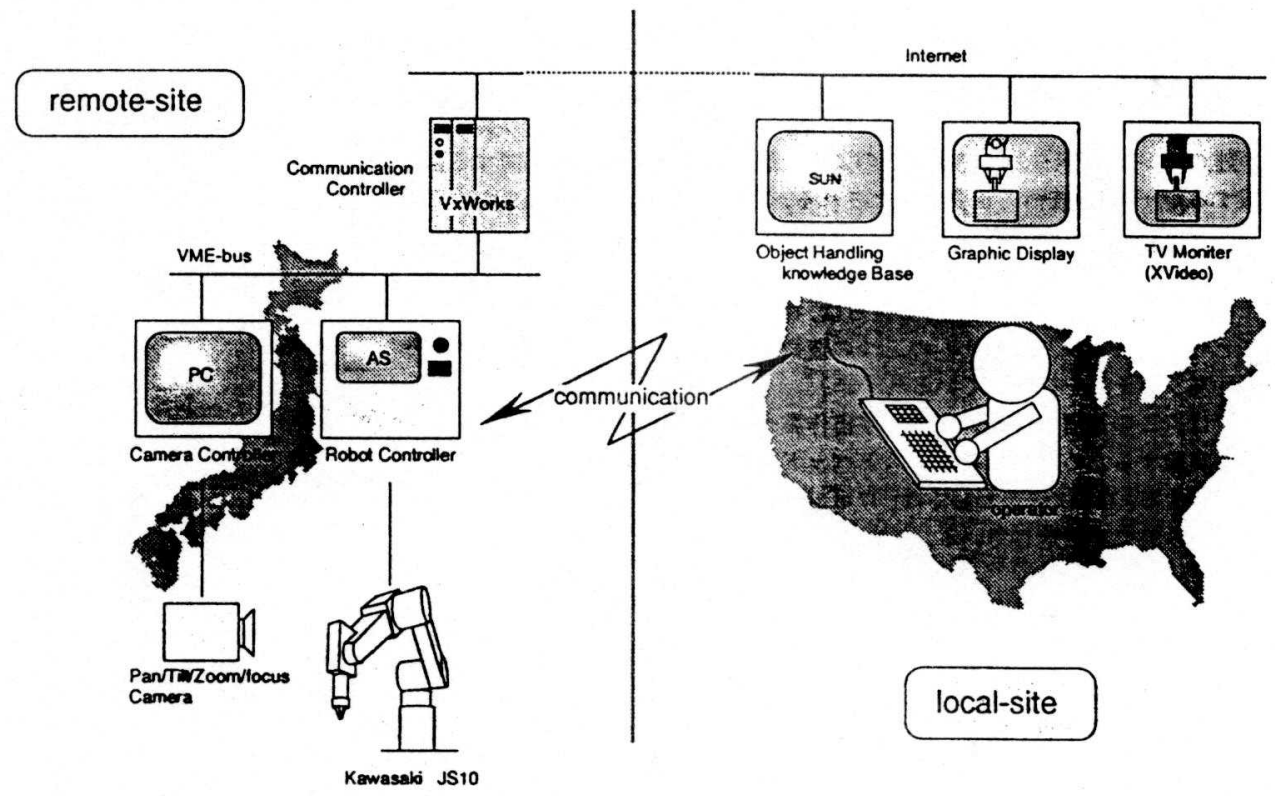

Figure 1 System block diagram 


\section{EXPERIMENT[2][3]}

To show the effectiveness of telerobotics system described in the chapter 2,3. The operator of the local site at JPL in USA operated the remote robot of the remote site at ETL in JAPAN to assemble the space structure.

\subsection{EXPERIMENT CONTENTS}

The space structure assembly experiment contents of super long distance telerobotics is as follows:

- DEPLOYMENT OF THE TRUSS STRUCTURE

The remote robot deployed the folded truss structure. The truss had a two-cell with a simple and passive latch.

- SETTING OF AN ORU HOLDER

The remote robot set an ORU holder on the truss structure. For setting, the ORU holder had to be turned and slided on the truss beam.

- INSTALLATION OF AN ORU

The remote robot grasped an ORU placed on appointed position, and placed it on the ORU holder.

- OPERATING OF TEACH MODE

To grasp and place the ORU which was shifted $5 \mathrm{~cm}$ from the appointed position intentionally, An local site operator changed to teach mode and operated remote robot directly.

\subsection{EXPERIMENT RESULTS}

Total time of space structure assembly experiment is shown in table 1. An experiment time took about one hour. Image transmission time rate and test bed assembly time rate was $18 \%$, $47 \%$ respectively against total time. Operator's decision time rate is $11 \%$ against total time. Thus we considered that human operation could be reduced by using knowledge base for regular operation. A rate of model matching time between remote site and local site is $10 \%$ against total time. Generally, it takes more time for data communication under super long distance telerobotics. By using higher level commands, remote site knowledge base and intelligent monitoring system was effective. Thus we considered that data communication and total operation time was reduced.

The assembly experiment completed without a interruption, though there is a position difference about 15 20 mm between an environment model and an real model. We confirmed that experiment system using a hybrid compliance/force control system had an allowance such as a position difference.

And an operator could correct a position or recover an operational error by using teach mode. Thus we confirmed that the teach mode which enable human to intervene to system was adaptable for an unexpected event.

\section{CONCLUSION}

In this paper, we have shown the telerobotics, system construction and MITI/NASA cooperation experiment of space structure assembly that be supposed space platform on-orbit.

We will examine and improve the telerobotics system as a follows:

- Addition of time lag simulation function

- Practice of various task experiments 
- Telemetry data sending when the remote system is executing some sub task

- Improvement of man-machine interface

We will study telerobotics in future and hope to cooperate with the international telerobotics development.

TABLE 1. Experiment results on space structure assembly

\begin{tabular}{|l|l|c|l|l|l|}
\hline $\begin{array}{l}\text { SUBTASK } \\
\text { NAME }\end{array}$ & $\begin{array}{l}\text { TOTAL } \\
\text { TIME }\end{array}$ & $\begin{array}{l}\text { IMAGE } \\
\text { TRANSMISSION }\end{array}$ & $\begin{array}{l}\text { MODEL } \\
\text { MATCHING }\end{array}$ & $\begin{array}{l}\text { TESTBED } \\
\text { ASSEMBLY }\end{array}$ & $\begin{array}{l}\text { OPERATOR } \\
\text { DECISION }\end{array}$ \\
\hline TRUSS 1 & $12^{\prime} 03^{\prime \prime}$ & $2^{\prime} 35^{\prime \prime}$ & $1^{\prime} 08^{\prime \prime}$ & $4^{\prime} 56^{\prime \prime}$ & $1^{\prime} 51^{\prime \prime}$ \\
\hline $\begin{array}{l}\text { TRUSS } 2 \\
\text { HOLUER }\end{array}$ & $12^{\prime} 02^{\prime \prime}$ & $2^{\prime} 08^{\prime \prime}$ & $1^{\prime} 37^{\prime \prime}$ & $5^{\prime} 00^{\prime \prime}$ & $1^{\prime} 38^{\prime \prime}$ \\
\hline ORU & $14^{\prime} 37^{\prime \prime}$ & $2^{\prime} 29^{\prime \prime}$ & $1^{\prime} 22^{\prime \prime}$ & $8^{\prime} 16^{\prime \prime}$ & $1^{\prime} 11^{\prime \prime}$ \\
\hline TOTAL & $53^{\prime} 30^{\prime \prime}$ & $2^{\prime} 50^{\prime \prime}$ & $1^{\prime} 02^{\prime \prime}$ & $7^{\prime \prime} 44^{\prime \prime}$ & $1^{\prime} 33^{\prime \prime}$ \\
\hline
\end{tabular}

\section{ACKNOWLEDGEMENTS}

KHI's authors appreciate ETL and JPL researchers gave us many constructive supports for this work.

\section{REFERENCES}

[1]. K.Machida et al., "Study on Long Distance Space Telerobotics: Concept of Interoperation Experiment between Japan and USA," Proc. 37th Space Sciences and Technology Conf., 1993(in Japanese).

[2]. K.Machida et al., "Study on Super Long Distance Telerobotics: Experiment of In-Orbit Platform Assembling," Proc. 38th Space Sciences and Technology Conf., 1994(in Japanese). [3]. K.Machida et al., "Study on Super Long Distance Telerobotics: Experiment of Platform Assembling from USA," Proc. 39th Space Sciences and Technology Conf., 1995 (in Japanese).

[4].S.Hirai et al., "Application of Intelligent Telerobotics to Super Long Distance Teleoperation ," JSME Annual Conf. on Robotics and Mechatronics 94, 1994(in Japanese).

[5]. F. Yasumoto et al., "Application of Super Long Distance Telerobotics to Space Structure Assembly Task," JSME Annual Conf. on Robotics and Mechatronics 95, 1995 (in Japanese). [6]. T.Katsuragawa et al., "Application of Hybrid Compliance/Force Control for Industrial Robot," JRSJ, vol.12, No.6, 1994 (in Japanese).

[7]. Y.Wakita et al., "Intelligent Monitoring System Applied to Super Long Distance Telerobotic Task," i-SAIRAS'94, 1994 (in English).

[8]. M.Kitami et al., "Telerobotics Test Bed for Space Structure Assembly," i-SAIRAS'95, 1995 (in English).

[9]. S.Hirai et al., "Object Model for Telerobot," Proc. IROS'88, 1988(in English).

[10]. Y.Wakita et al., "Automatic Camera-work Control for Intelligent Motoring of Telerobotic Tasks," Proc. IROS'92,1992(in English).

[11]. T.Matsui et al., "EusLisp Reference Manual," ETL-TR-95-19, ETL, 1995. (in Japanese). 